\title{
Design of Multiobjective Optimal Laser Ablation Parameters of Hole Opening in Package-on-package Process
}

\author{
Jian-Long Kuo* \\ Department of Mechatronics Engineering, National Kaohsiung University of Science and Technology, \\ No. 2, Zhuoyue Rd. Nanzih, Koahsiung 811, Taiwan
}

(Received April 9, 2020; accepted September 8, 2020)

Keywords: multiobjective optimal design, package-on-package, laser ablation, hole opening

In this paper, the design of the multiobjective optimal parameters of the IC package FCPOPCSP (Flip Chip Package on Package Chip Scale Package) manufacturing process was studied. In the IC package FCPOPCSP manufacturing process, the laser ablation of hole openings is the key process affecting the product yield rate of flip chips in the IC package product. Two hole opening problems related to the opening hole diameter (OHD) and inside hole diameter (IHD) were selected as objective functions. The nominal-the-best optimization problem was examined in the studied quality engineering. A statistical model was provided to study the optimization problem of the laser ablation machine. First, the dual response surface method was used to formulate the statistical model. Three laser ablation machine parameters, namely, laser power, draw step, and step period, were selected as three control factors. The fuzzy multiple performance characteristics index (MPCI) method was used to combine the two objective functions into one integrated index. Lastly, the orthogonal particle swarm optimization (OPSO) method was used to solve the nonlinear statistical problem. The derived optimal solution can provide the optimal parameter settings for laser ablation machines in practical industrial applications. The product yield rate can be improved by adopting the nominal-the-best solution set. The suggested model can be further uploaded to be a cloud model on a cloud server to provide the optimal manufacturing strategy of the manufacturing process in the IC package industry. A smart manufacturing function can be achieved to fulfill the modern Internet of Things and Industry 4.0 applications.

\section{Introduction}

In this work, the optimal design in semiconductor manufacturing processes related to the flip chip package technology FCPOPCSP (Flip Chip Package on Package Chip Scale Package) was studied. The ablation machine parameter settings in the manufacturing process greatly impact the product quality. Laser processing and laser ablation are used to create holes and remove the sealing film adhesive so as to reveal the solder balls providing the next product stacking process. The technology of laser ablation applied to the removal of the sealing film adhesive is still new. Therefore, setting the machine parameters is a challenging technical 
problem. Also, laser ablation is a manufacturing process that cannot be redone. If hole opening fails, the product itself might be damaged and therefore fail.

Dreiza et al. $^{(1)}$ and Zwenger et al. ${ }^{(2)}$ studied the warpage problem of IC packaging products. Warpage might lead to the failure of stack welding. Some improvement method should be provided to overcome this problem. The design book $^{(3)}$ specifies the standards JEDEC JC-11 and JC-16. JEDEC JC-11 describes the stacked package-on-package (PoP) design and the mechanical structure. JEDEC JC-16 describes the standard of the top-layer memory pin-out. However, technical literature seldom mentions the practical manufacturing problem of hole opening.

In addition to the warpage problem, the product stack processing might be affected by the size and position of the open hole. The open hole size is demanded to be smaller year by year. The distance between solder balls is also becoming narrower. How to create a good open hole has become a challenging technical problem. Galantucci ${ }^{(4)}$ developed a 3D numerical model of laser ablation. The ablation depth is determined by the machining surface and the laser energy density. The laser energy density is determined by the laser inherent power and the laser machining time.

Choi et al. ${ }^{(5)}$ suggested a hole area modulation (HAM) method to determine the optimal laser ablation parameters, such as the moving velocity of the mask, the hole-to-hole distance, and the number of repetitions of machining. In this work, we used a laser drill and studied three control factors for optimizing the design: laser power, laser draw step, and laser step period. The dual-objective design problem was studied. The two design objectives are to observe the laser drill depth and to fabricate the bottom-hole aperture by laser drilling.

\section{PoP Technology}

Two or more devices can be integrated by PoP technology such as vertical stacking or the back carrying method. ${ }^{(6)}$ Electronic wires can be connected directly. The PCB can be connected to bottom devices. In recent years, PoP technology has been applied to the 3D integration of logic and memory units in mobile handsets and other portable multimedia devices. ${ }^{(7)}$

As shown in Fig. 1, the logic circuit or microcontroller unit is at the bottom and the memory unit is on top. In the welding process of PoP technology, the factors that possibly affect the quality of welding are the welding surface size, open hole size, open hole position, welding flux, solder ball size, and substrate warpage. ${ }^{(8)}$

As shown in Fig. 2, substrate warpage and solder ball quality might cause the nonwetting problem of solder balls. A change in open hole size or open hole position might cause the bridge problem of solder balls. ${ }^{(9)}$ To maintain the optimal quality of the manufacturing process, a good method of parameter control is required to keep the open hole size constant.

In the flip chip package product, there are material units such as the die, bump, underfill, and compound. Differences among the coefficient of thermal expansion (CTE) of material units might lead to warpage. As one solution to this problem, the whole product is covered with an adhesive sealing film to avoid the warpage phenomenon. Therefore, the adhesive sealing film on the top solder balls must be removed in the packaging process. 

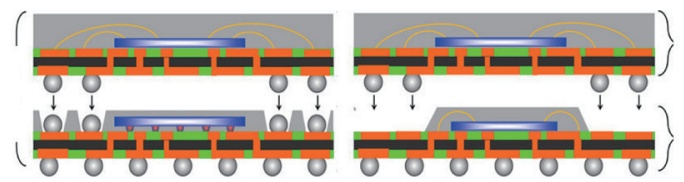

Fig. 1. (Color online) The logic circuit or microcontroller is at the bottom and the memory unit is on top. The logic circuit can be a wire-bonding or flip chip package product.

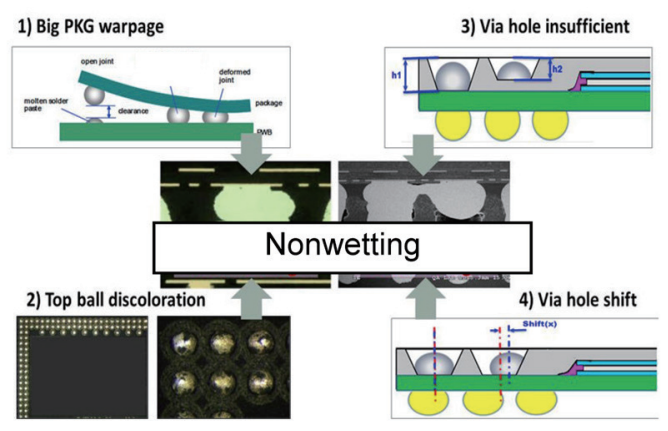

Fig. 2. (Color online) Poor open hole quality might cause stacking failure in the product.

The open hole size of the top solder balls is defined as the sum of the volumes of the toplayer and bottom-layer solder balls, as shown in Fig. 3.

$$
\frac{4}{3} \pi r_{1}^{3}+\frac{1}{2}\left(\frac{4}{3} \pi r_{2}^{3}\right)=\frac{\pi h}{3}\left(R^{\prime 2}+R^{\prime} r^{\prime}+r^{\prime 2}\right)
$$

$r_{1}$ : radius of top-layer solder balls

$r_{2}$ : radius of bottom-layer solder balls

$h$ : hole depth (HD) in bottom layers

$R^{\prime}$ : upper radius of hole in bottom layer

$r$ : lower radius of hole in bottom layer

By calculating the associated geometric sizes, the required open hole size can be specified. However, in practice, the open hole size might have a mechanical size tolerance problem. Also, the practical process requires the use of solder paste material, the heat expansion of which leads to the problem of insufficient hole volume when welding.

Therefore, the hole volume is enlarged by $25 \%$ of the ideal volume to ensure sufficient space. The modified equation is written as

$$
1.25\left\{\frac{4}{3} \pi r_{1}^{3}+\frac{1}{2}\left(\frac{4}{3} \pi r_{2}^{3}\right)\right\}=\frac{\pi h}{3}\left(R^{\prime 2}+R^{\prime} r^{\prime}+r^{\prime 2}\right) .
$$

In this paper, the design sizes are specified and listed in Table 1. Then, the final minimal hole depth can be derived as

$$
h=\frac{3.75}{\pi\left(R^{\prime 2}+R^{\prime} r^{\prime}+r^{\prime 2}\right)}\left\{\frac{4}{3} \pi r_{1}^{3}+\frac{1}{2}\left(\frac{4}{3} \pi r_{2}^{3}\right)\right\} .
$$



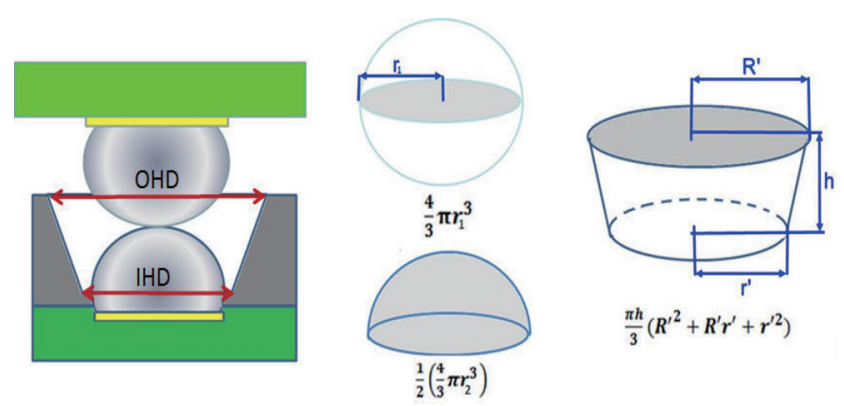

Fig. 3. (Color online) Relationship between open hole size and top-layer and bottom-layer solder balls.

Table 1

Flip chip product geometric size.

\begin{tabular}{cccc}
\hline $\begin{array}{c}r_{1}: \text { radius of top-layer } \\
\text { solder balls }\end{array}$ & $\begin{array}{c}r_{2}: \text { radius of bottom-layer } \\
\text { solder balls }\end{array}$ & $\begin{array}{c}R^{\prime}: \text { upper radius of hole } \\
\text { in bottom layer }\end{array}$ & $\begin{array}{c}r^{\prime}: \text { lower radius of hole } \\
\text { in bottom layer }\end{array}$ \\
\hline $0.1625 \mathrm{~mm}$ & $0.1625 \mathrm{~mm}$ & $0.285 \mathrm{~mm}$ & $0.19 \mathrm{~mm}$ \\
\hline
\end{tabular}

The minimal depth is defined as $0.19 \mathrm{~mm}$. The tolerance of the measuring machine is $30 \mu \mathrm{m}$. Therefore, $0.19 \pm 0.03 \mathrm{~mm}$ is specified to be the nominal value for nominal-the-best optimization. The upper diameter of the open hole is $570 \mu \mathrm{m}$ and the lower diameter of the open hole is $380 \mu \mathrm{m}$. Three different depths, 170, 190, and $210 \mu \mathrm{m}$, are compared, as shown in Fig. 4 and Table 2. The bridge problem occurred when the depth was $170 \mu \mathrm{m}$.

\subsection{Flip chip manufacturing process}

In flip chip technology, the die surface is connected to the underlying substrate using metal conductors. ${ }^{(10)}$ The metal conductor can be a metal bump, tape for automated bonding, anisotropic conductive adhesive, polymer bump, or wire bond. ${ }^{(11)}$ Among them, the metal bump is the main trend in flip chip technology. In this study, the solder bump is used as the metal bump. The manufacturing process resembles SMT technology. The chip is welded at the welding area at a high temperature.

Underfill is used to protect the solder bump. If the product has the FCCSP structure, the molding process is added after the underfill process. The FCPOPCSP standard manufacturing process is shown in Fig. 5 and involves (1) bumping, (2) wafer grinding, (3) wafer sawing, (4) flip chip bonding, (5) reflow, (6) flux cleaning, (7) underfilling, (8) curing, (9) top-side ball mounting, (10) reflow, (11) cleaning, (12) molding, (13) laser ablation, (14) laser marking, (15) bottom ball mounting, (16) singulation, (17) visual inspection, and (18) packaging. ${ }^{(12)}$ The cross section of the finished product is shown in Fig. 6.

The key technology in the FCPOPCSP manufacturing process is laser ablation using a laser drill. In laser ablation, the material surface is irradiated with a laser of high energy density to open a hole. The size and quality of the open hole depend on the warpage of the product and the energy density of the laser. The energy density is determined by the laser power, moving velocity, stopping time, and working time. 


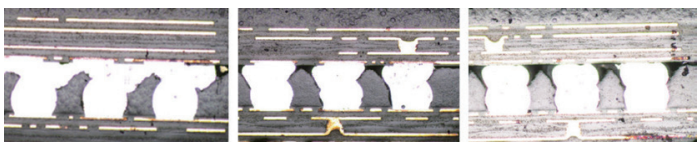

Fig. 4. (Color online) Stacking results for open HDs of 170,190 , and $210 \mu \mathrm{m}$.

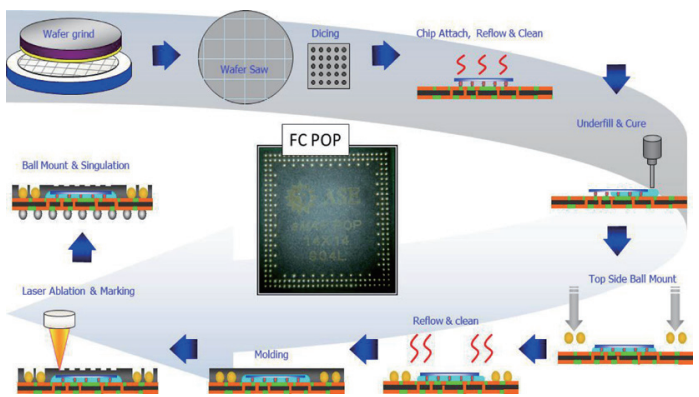

Fig. 5. (Color online) FCPOPCSP product manufacturing process.
Table 2

Flip chip product open hole geometric size.

\begin{tabular}{ccc}
\hline $\begin{array}{c}\text { Upper radius of } \\
\text { hole in bottom } \\
\text { layer }(\mu \mathrm{m})\end{array}$ & $\begin{array}{c}\text { Lower radius of } \\
\text { hole in bottom } \\
\text { layer }(\mu \mathrm{m})\end{array}$ & HD $(\mu \mathrm{m})$ \\
\hline 570 & 380 & 170 \\
570 & 380 & 190 \\
570 & 380 & 210 \\
\hline
\end{tabular}

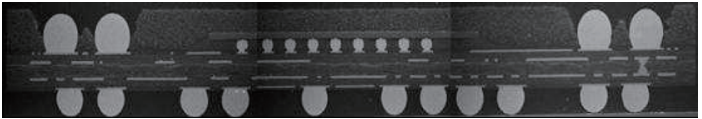

Fig. 6. (Color online) Cross section of finished FCPOPCSP product.

In this study, the above laser parameters were studied to obtain the optimal product yield rate. The dual response surface method is used to formulate a statistical model for the studied problem.

\subsection{Laser drill machine}

The laser drill machine is made of a pulse fiber laser, as shown in Fig. 7, and has a high energy conversion rate, high-quality light beam, low cost, and long life. ${ }^{(13)}$ There are two laser sources. Each laser source is split into two light beams. Therefore, there are a total of four light beams to perform drilling.

The structure of the drill machine is shown in Figs. 8 and 9. ${ }^{(14)}$ The laser module provides a $30 \mathrm{~W}$ laser source that is split into two $15 \mathrm{~W}$ laser beams by beam splitters. Then, each laser beam passes through a beam expander telescope (BET), scanner, and F-theta lens. Finally, high-energy-density laser beams act as laser drills at the worktable. ${ }^{(15)}$

\subsection{Laser drilling process}

According to the manual, the parameters of pulse-type lasers, such as laser power, laser frequency, laser pattern, draw step, and step period, are provided to operators for setting up the devices. To simplify the assessment of parameters, the following three control factors are selected and their effects on open hole quality are studied: laser power, draw step, and step period. Other factors such as laser frequency and laser pattern are kept unchanged.

As shown in Fig. 10, the machine parameters are described as follows: (a) draw step: distance of laser movement from the current operating point A to the next point B; (b) step period: time 


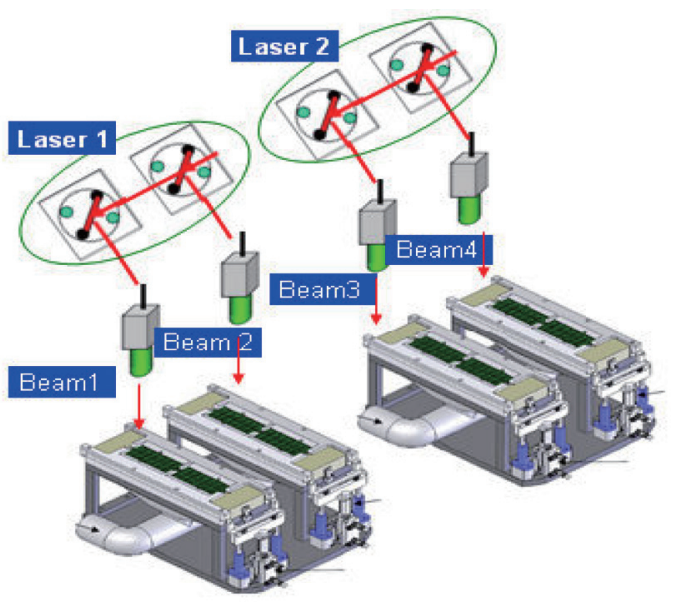

Fig. 7. (Color online) Two laser sources are split into four light beams.

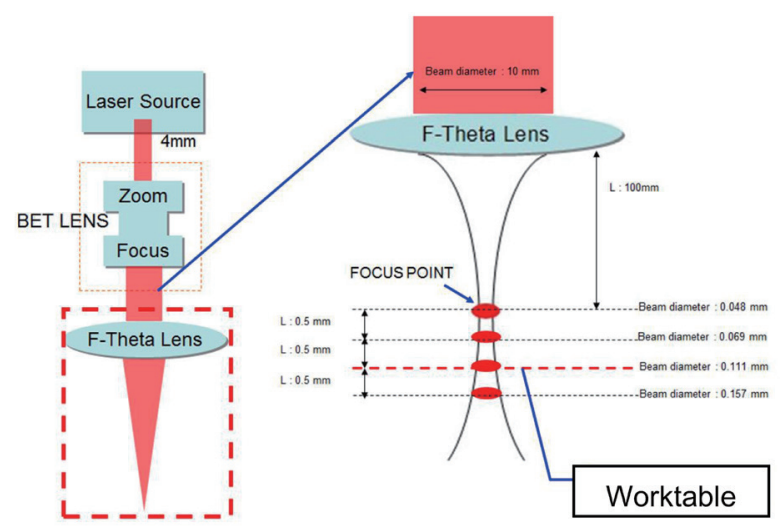

Fig. 9. (Color online) Structure of laser drill machine.

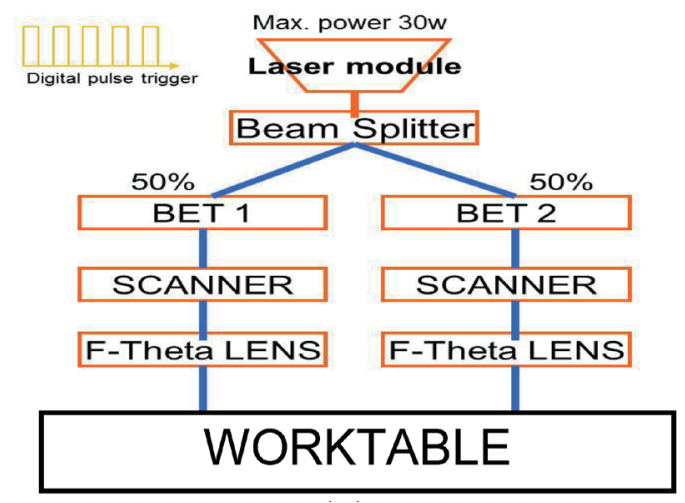

(a)

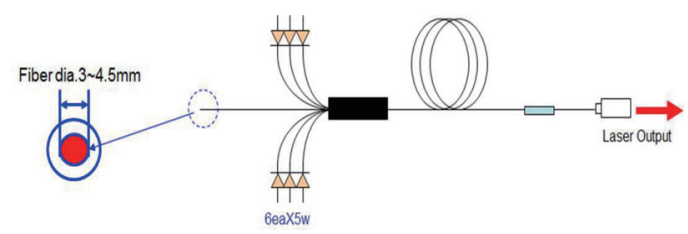

(b)

Fig. 8. (Color online) Flow of laser beam in laser drill machine.

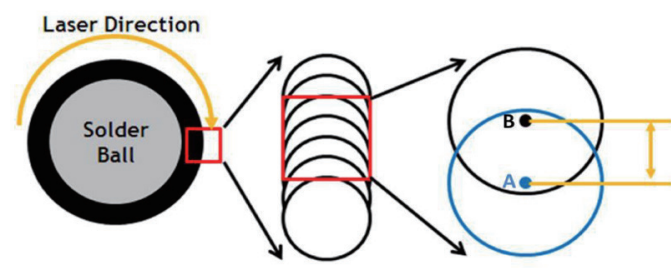

Fig. 10. (Color online) Schematic of draw step and step period.

interval at which the laser moves from the current operating point $\mathrm{A}$ to the next point $\mathrm{B}$. Table 3 shows the three control factors selected for study: laser power (power), draw step, and step period.

Three laser patterns are used in drilling and polishing, as shown in Figs. 11 and 12. Pattern 1: five different concentric circles are used to determine the size of the open hole and remove most of the sealing film adhesive. Pattern 2: six different concentric circles are used to remove the remaining sealing film adhesive and reshape the opening hole. Pattern 3: six different concentric circles are used to clean the remained sealing film adhesive on the surface of solder balls. 
Table 3

Parameter ranges for laser ablation.

\begin{tabular}{lc}
\hline Parameter & Range \\
\hline Power $(\mathrm{W})$ & $9 \pm 0.5$ \\
Draw step $(\mu \mathrm{m})$ & $18 \pm 5$ \\
Step period $(\mu \mathrm{s})$ & $20 \pm 5$ \\
\hline
\end{tabular}

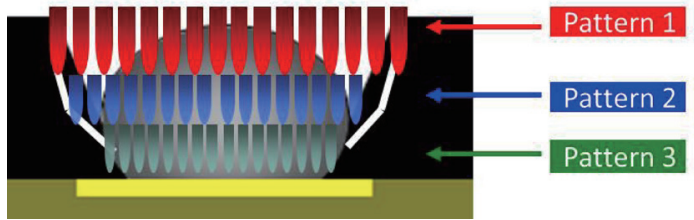

Fig. 11. (Color online) Laser patterns used in drilling.

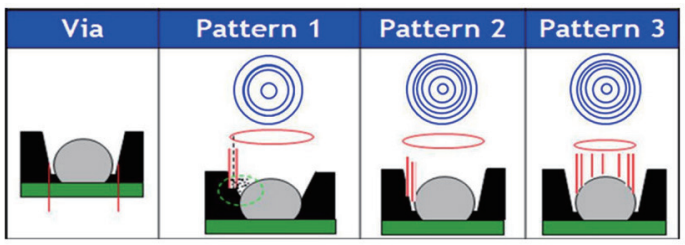

Fig. 12. (Color online) Three patterns used for laser ablation.
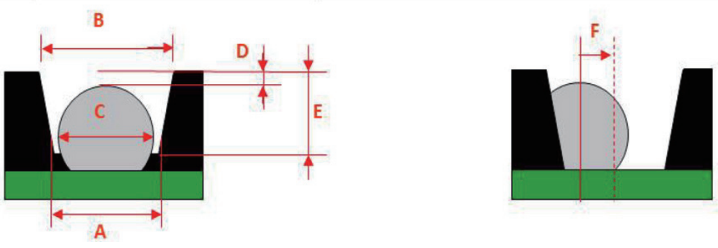

Fig. 13. (Color online) Open hole size parameter definitions.

The quality of the open hole can be assessed by observing and examining the following six size specifications (A to F) shown in Fig. 13: (A) the inside hole diameter (IHD) for the bottom layer; (B) the outside hole diameter for the top layer, OHD; (C) the inner ball diameter (IBD) exposed; (D) top ball depth (TBD); (E) hole depth (HD); and (F) hole shift (SR).

OHD and SR depend on the laser positioning precision. IBD depends on the size of the mount ball. TBD depends on the size of solder balls. The OHD and IHD are strongly affected by parameters of the laser drilling machine. Therefore, the objective functions of HD and IHD are selected to study the dual-objective optimization problem.

\section{Experimental Test Cases}

Two experimental setups are shown in Fig. 14. One is the laser drill machine (EO Technics) that performs the major drilling and polishing functions. The other is the laser profile scanner (CyberOptics) used to measure the size of the open hole to examine the open hole quality.

Sensors and their related materials and technology are described below.

a) Laser drill machine: infrared (IR) viewers and related materials and technology

IR viewers are embedded in the laser drill machine. These IR viewers have been designed to observe the indirect radiation of an IR laser via high-performance image conversion. Each IR viewer has an electrostatic focusing system based on a high-grade image converter. The IR viewer can focus the emitted or reflected light from a subject into an image tube where an electron image is generated. When an acceleration voltage of $16-18 \mathrm{kV}$ is used to display the electron image on the output phosphor screen, the fluorescent green light output at $550 \mathrm{~nm}$ is observed via an adjustable eyepiece lens.

b) Laser profile scanner: 3D laser profile sensor and related materials and technology

The profile sensors have compact size and light weight. The controllers of the profile sensors are integrated in the housing, simplifying the cabling arrangement and mechanical 


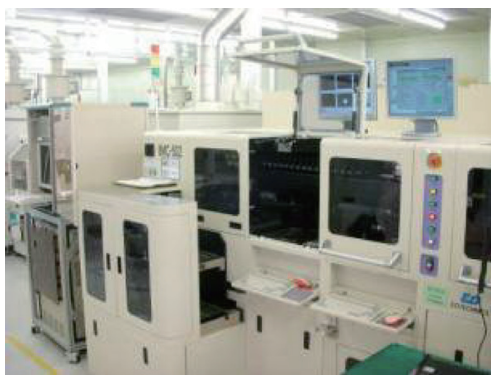

(a)

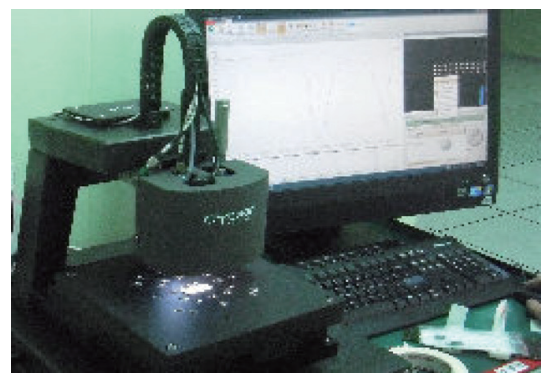

(b)

Fig. 14. (Color online) Experimental setups. (a) Laser drill machine. (b) Laser profile scanner.

integration. The laser profile sensors are suitable for dynamic measurement tasks owing to their compact size and high profile frequency. These profile scanners are widely used in production line control and robot mounting processes.

As shown in Table 4, the objective functions of HD and IHD are selected to study the optimization problem. In Case 1, the objective function of HD is studied. In Case 2, the objective function of IHD is studied. In Case 3, the two objective functions are integrated into one compact multiple performance characteristics index (MPCI) by the fuzzy method. Because of the page limit, we concentrate on Case 3. In Cases 1 and 2, the same principle is used to obtain the objective functions. To simplify the description, the discussions of Cases 1 and 2 are omitted here.

As shown in Fig. 15, three control factors are selected as studied variables: laser power, draw step, and step period. Three levels of values are defined to investigate the possible variation range of the parameters. The optimal value can be found in the defined range by the response surface method based on the first-order statistical model. To overcome the nonlinearity of the derived statistical model, the orthogonal particle swarm optimization (OPSO) method is adopted to find the optimal solution. In this paper, the response surface method is used to formulate a statistical model. ${ }^{(16)}$

To convert the measured value from nominal-the-best to larger-the-better, the following equation is defined:

$$
y_{m}=\frac{1}{\left(y_{i}-m\right)^{2}} \text {. }
$$

Here, $m$ is the target value, which is set to $220 \mu \mathrm{m}$. The squared term is used to consider the possible positive or negative conditions that could occur. Therefore, $y_{m}$ is considered to be always positive here.

\subsection{Fuzzy inference with MPCI in statistical model}

There are two objective functions, HD and IHD, in the studied problem. To integrate the two objective functions into one compact function, the MPCI method is adopted. The two objective functions can be defined as larger-the-better problems and are regarded as two inputs 
Table 4

Definitions of objective functions for the nominal-the-best study.

\begin{tabular}{lcc}
\hline Case & Method & $\begin{array}{c}\text { Nominal-the-best } \\
\text { value }\end{array}$ \\
\hline Case 1 & $\begin{array}{c}\text { Response surface } \\
\text { method }\end{array}$ & HD $=220 \mu \mathrm{m}$ \\
\hline Case 2 & $\begin{array}{c}\text { Response surface } \\
\text { method }\end{array}$ & IHD $=380 \mu \mathrm{m}$ \\
\hline Case 3 & $\begin{array}{c}\text { Fuzzy-MPCI-based } \\
\text { response surface } \\
\text { method }\end{array}$ & $\begin{array}{c}\text { HD }=220 \mu \mathrm{m} \& \\
\text { IHD }=380 \mu \mathrm{m}\end{array}$ \\
\hline
\end{tabular}

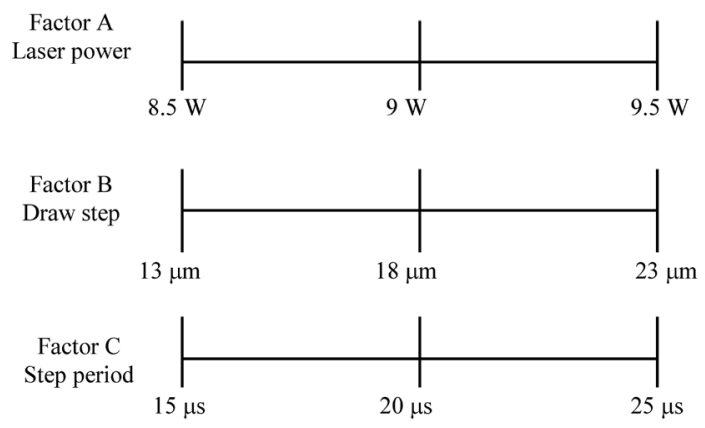

Fig. 15. Level and range definitions of three control factors.

for the fuzzy inference process that ultimately outputs the MPCI value. The fuzzy set for the specified objective function is defined as the membership function

$$
A_{i}=\left\{x, \mu_{A_{i}}(x) \mid x \in U\right\},
$$

where $U$ is the overall range of the corresponding fuzzy set $A_{i} . \mu_{A i}{ }^{(x)}$ is the membership function of $A_{i}, i=1,2,3$.

As shown in Figs. 16 and 17, the precise values of HD and IHD are fuzzified in the three fuzzy meanings, small (S), medium (M), and large (L). Triangular functions are defined to represent the three fuzzy meanings. As shown in Fig. 18, the MPCI value is also fuzzified in five fuzzy meanings. Therefore, fuzzy inferences among the fuzzy meanings are classified as in Table 5.

In this paper, the Mamdani inference method is adopted. For example,

$$
\begin{aligned}
& R_{1}: \text { if } x_{1} \text { is } A_{1} \text { and } x_{2} \text { is } B_{1} \text {, then } y \text { is } C_{1} . \\
& R_{2}: \text { if } x_{1} \text { is } A_{2} \text { and } x_{2} \text { is } B_{2} \text {, then } y \text { is } C_{2} . \\
& R_{3}: \text { if } x_{1} \text { is } A_{3} \text { and } x_{2} \text { is } B_{3} \text {, then } y \text { is } C_{3} .
\end{aligned}
$$

Here, $\mu_{A i}, \mu_{B i}$, and $\mu_{C i}$ are the respective membership functions of $A_{i}, B_{i}$, and $C_{i}$.

One of the fuzzy outputs is illustrated as

$$
\begin{aligned}
\mu_{C_{0}}(y)= & {\left[\mu_{A_{1}}\left(x_{1}\right) \wedge \mu_{B_{1}}\left(x_{2}\right) \wedge \mu_{C_{1}}(y)\right] } \\
& \vee\left[\mu_{A_{2}}\left(x_{1}\right) \wedge \mu_{B_{2}}\left(x_{2}\right) \wedge \mu_{C_{2}}(y)\right] \vee\left[\mu_{A_{3}}\left(x_{1}\right) \wedge \mu_{B_{3}}\left(x_{2}\right) \wedge \mu_{C_{3}}(y)\right] .
\end{aligned}
$$

The fuzzy rules defined in Table 5 are given in greater detail. In the fuzzy inference process, the following fuzzy meanings and logic inference process are defined. The relationships between two objective function inputs and fuzzy inference relations are described as shown in Table 5. 
membership

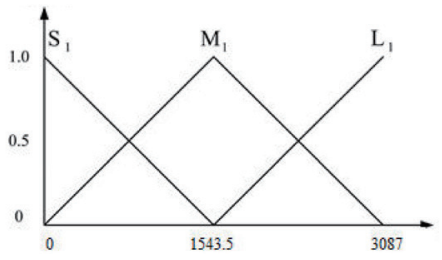

SN ratio

Fig. 16. Membership function of nominal HD value.

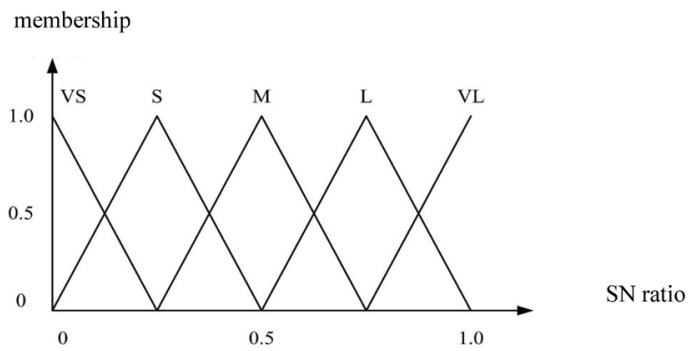

membership

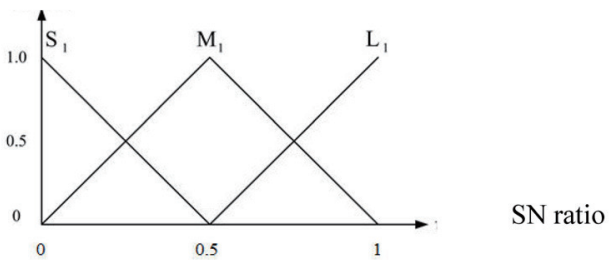

Fig. 17. Membership function of nominal IHD value.

Table 5

Fuzzy inference table.

\begin{tabular}{|c|c|c|c|c|}
\hline \multirow{2}{*}{\multicolumn{2}{|c|}{ Output C (MPCI) }} & \multicolumn{3}{|c|}{ Input A } \\
\hline & & $\mathrm{S}$ & M & $\mathrm{L}$ \\
\hline \multirow{3}{*}{ Input $\mathrm{B}$} & $\mathrm{S}$ & VS & S & M \\
\hline & $\mathrm{M}$ & $\mathrm{S}$ & M & $\mathrm{L}$ \\
\hline & $\mathrm{L}$ & $\mathrm{M}$ & $\mathrm{L}$ & VL \\
\hline
\end{tabular}

Fig. 18. Membership function of MPCI value.

$R_{1}$ : if $A$ is small and $B$ is small, then $C$ is very small.

$R_{2}:$ if $A$ is small and $B$ is medium, then $C$ is small.

$R_{9}:$ if $A$ is large and $B$ is large, then $C$ is very large.

Finally, defuzzification is adopted to integrate the nine values from nine fuzzy rules. The weighted-average method is selected as the defuzzification method.

$$
y^{*}=\frac{\sum_{i=1}^{L} \mu_{c}\left(y_{i}\right) y_{i}}{\sum_{i=1}^{L} \mu_{c}\left(y_{i}\right)}
$$

Here, $\mu_{c}\left(y_{i}\right)$ is the membership function of $y_{i}$ belonging to the fuzzy set $C . y^{*}$ is defined as the MPCI. The overall MPCI inference process is summarized in Fig. 19.

Before running the fuzzy inference, membership functions are defined. The membership functions of HD and IHD are defined to have three levels of fuzzy meanings: S, M, and L. Triangular functions are selected as membership functions, as shown in Figs. 9 and 10.

For the MPCI value, a normalized value is defined. As shown in Fig. 11, there are five levels defined in the fuzzy meaning: very small (VS), S, M, L, and very large (VL). The membership functions of HD and IHD are defined as two input fuzzy meanings. The entire fuzzy inference process is described in Table 5. The final MPCI values output by fuzzy inference are normalized values, as shown in Tables 6 and 7. 


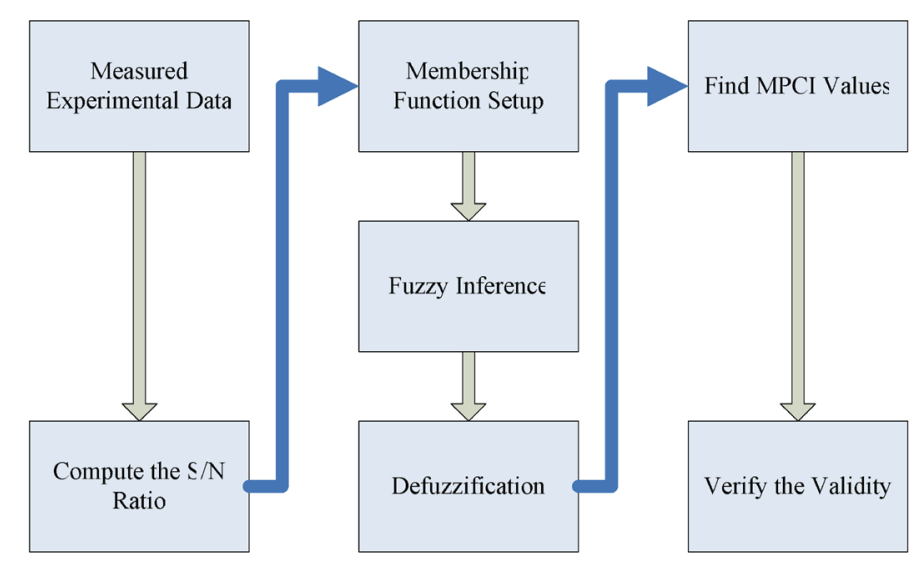

Fig. 19. (Color online) MPCI fuzzy inference process.

Table 6

Measured $y_{m}$ of 16 experimental runs for three cases.

\begin{tabular}{ccc}
\hline HD & IHD & MPCI \\
\hline 0.05516 & 0.04201 & 0.02103 \\
0.00160 & 0.02407 & 0.01203 \\
0.00014 & 0.28054 & 0.14027 \\
0.00018 & 0.44563 & 0.22282 \\
0.00006 & 0.03345 & 0.01672 \\
0.00004 & 0.11704 & 0.05852 \\
0.00211 & 0.02998 & 0.01499 \\
0.01911 & 0.66314 & 0.33158 \\
0.05403 & 0.18388 & 0.09196 \\
0.00158 & 0.59354 & 0.29677 \\
0.00014 & 0.20272 & 0.10136 \\
0.00018 & 0.05513 & 0.02756 \\
0.00006 & 0.10299 & 0.05150 \\
0.00004 & 0.04774 & 0.02387 \\
0.00206 & 0.09013 & 0.04763 \\
0.01869 & 0.79435 & 0.39718 \\
\hline
\end{tabular}

Table 7

$y_{m}$ data of four central-point experimental runs for three cases.

\begin{tabular}{lcc}
\hline \multicolumn{1}{c}{ HD } & IHD & MPCI \\
\hline 0.4005768 & 0.03384 & 0.03459 \\
0.30864197 & 0.66206 & 0.83087 \\
0.5827166 & 0.20327 & 0.12083 \\
0.91827365 & 0.06422 & 0.19498 \\
\hline
\end{tabular}

In accordance with the standard definition of the response surface method, the combinational table has three control factors and two noise factors. The 16 combinational sets in Table 6 are required to perform the experimental runs. To verify the curvature of the experimental data, four central-point experimental runs are performed in Table 7. Experimental runs were each repeated four times.

In Case 3, both HD and IHD are considered at the same time by fuzzy inference and integrated into one compact MPCI value to derive the optimal solution of the dual objective functions.

\subsection{Response surface formulation}

The results of 16 experimental runs are listed in Table 6, which is called the combinational table in the response surface method. The results of four central-point experimental runs are 
listed in Table 7. The average MPCI value of the four central-point experimental data is derived as

$$
\bar{y}_{c}=(0.03459+0.83087+0.12083+0.19498) / 4=0.29532 \text {. }
$$

The average MPCI value of 16 experimental runs is derived as

$$
\begin{aligned}
\bar{y}_{f}= & (0.02103+0.01203+0.14027+0.22282+0.01672 \\
& +0.05852+0.01499+0.33158+0.09196+0.29677+0.10136 \\
& +0.02756+0.05150+0.02387+0.04763+0.39718) / 16 \\
= & 0.11599 .
\end{aligned}
$$

The sum of curvature variance is calculated as

$$
S S_{C}=\frac{n_{f} n_{c}\left(\bar{y}_{f}-\bar{y}_{c}\right)^{2}}{n_{f}+n_{c}}=\frac{16 \times 4(0.11599-0.29532)^{2}}{16+4}=0.102910 .
$$

The sum of error variance is calculated as

$$
S S_{E}=\sum_{i=1}^{n_{c}}\left(y_{m}-\bar{y}_{c}\right)^{2}=0.395315 .
$$

The $F$ statistics value is obtained as

$$
F=\frac{S S_{c} / 1}{S S_{E} /\left(n_{c}-1\right)}=\frac{0.10291 / 1}{0.395315 /(4-1)}=0.780969
$$

The overall regressive model is described as

$$
\begin{aligned}
y= & Q_{1}+Q_{2} \times x_{1}+Q_{3} \times x_{2}+Q_{4} \times x_{3}+Q_{5} \times z_{1} \\
& +Q_{6} \times z_{2}+Q_{7} \times x_{1} x_{2}+Q_{8} \times x_{1} x_{3}+Q_{9} \times x_{2} x_{3} \\
& +Q_{10} \times x_{1} z_{1}+Q_{11} \times x_{2} z_{1}+Q_{12} \times x_{3} z_{1}+Q_{13} \times x_{1} z_{2} \\
& +Q_{14} \times x_{2} z_{2}+Q_{15} \times x_{3} z_{2} \\
= & 0.15185+0.05530 \times x_{1}+0.04444 \times x_{2}+0.00176 \times x_{3}+0.01374 \times z_{1} \\
& +0.02953 \times z_{2}+0.02906 \times x_{1} x_{2}+0.02973 \times x_{1} x_{3}+0.03566 \times x_{2} x_{3} \\
& +0.00131 \times x_{1} z_{1}-0.03073 \times x_{2} z_{1}-0.00145 \times x_{3} z_{1}+0.04300 \times x_{1} z_{2} \\
& -0.00587 \times x_{2} z_{2}-0.01673 \times x_{3} z_{2} .
\end{aligned}
$$

The mean response surface, which is considered to be the major objective function for optimization, is derived by the response surface method. 


$$
\begin{aligned}
E_{z}(y(x, z))= & b_{0}+x^{\prime} b+x^{\prime} B x \\
= & Q_{1}+Q_{2} \times x_{1}+Q_{3} \times x_{2}+Q_{4} \times x_{3} \\
& +Q_{7} \times x_{1} x_{2}+Q_{8} \times x_{1} x_{3}+Q_{9} \times x_{2} x_{3} \\
= & 0.15185+0.05530 \times x_{1}+0.04444 \times x_{2}+0.00176 \times x_{3} \\
& +0.02906 \times x_{1} x_{2}+0.02973 \times x_{1} x_{3}+0.03566 \times x_{2} x_{3}
\end{aligned}
$$

The variance of the response surface is also derived as

$$
\operatorname{Var}_{z}(y(x, z))=\sum\left(\partial y / \partial z_{i}\right)^{2} \sigma_{z i}^{2}=\left(\partial y / \partial z_{1}\right)^{2} \sigma_{z 1}^{2}+\left(\partial y / \partial z_{2}\right)^{2} \sigma_{z 2}^{2} .
$$

The partial derivatives of the $y$ value are derived as

$$
\begin{gathered}
\partial y / \partial z_{1}=0.01374+0.00131 \times x_{1}-0.03073 \times x_{2}-0.00145 \times x_{3}, \\
\partial y / \partial z_{2}=0.02953 \times z_{2}+0.04300 \times x_{1}-0.00587 \times x_{2}-0.01673 \times x_{3} .
\end{gathered}
$$

Assuming that the noise factors $z_{1}$ and $z_{2}$ vary when measuring the data, set the standard deviations $\sigma_{s 1}=1$ and $\sigma_{s 2}=1$.

$$
\begin{aligned}
\operatorname{Var}_{z}(y(x, z))= & \left(1.06110+2.57584 \times x_{1}-1.19134 \times x_{2}-1.02823 \times x_{3}\right. \\
& -0.58547 \times x_{1} x_{2}-1.44284 \times x_{1} x_{3}+0.28537 \times x_{2} x_{3} \\
& \left.+1.85046 \times x_{1}^{2}+0.97889 \times x_{2}^{2}+0.28213 \times x_{3}^{2}\right) \times 10^{-3}
\end{aligned}
$$

The variance response surface is set to be smaller than 100 to form an inequality of the constraint condition for optimization.

$$
\begin{aligned}
\operatorname{Var}_{z}(y(x, z))= & \left(\left(1.06110+2.57584 \times x_{1}-1.19134 \times x_{2}-1.02823 \times x_{3}\right.\right. \\
& -0.58547 \times x_{1} x_{2}-1.44284 \times x_{1} x_{3}+0.28537 \times x_{2} x_{3} \\
& \left.\left.+1.85046 \times x_{1}^{2}+0.97889 \times x_{2}^{2}+0.28213 \times x_{3}^{2}\right) \times 10^{-3}\right) \leq 100
\end{aligned}
$$

The respective ranges are defined as

$$
-1.0 \leq x_{1} \leq 1.0,-1.0 \leq x_{2} \leq 1.0,-1.0 \leq x_{3} \leq 1.0 .
$$

The final optimal solution $\left(x_{1}, x_{2}, x_{3}\right)=(1,1,1)$ is obtained. That means that the optimal values for the three control factors are a laser power of $9.5 \mathrm{~W}$, a draw step of $23 \mu \mathrm{m}$, and a step period of $25 \mu \mathrm{s}$. The optimal value of HD is $225.3621 \mu \mathrm{m}$. The optimal value of IHD is $385.3621 \mu \mathrm{m}$. The corresponding mean response surface is plotted in Fig. 20. The contour plot of the mean response surface is shown in Fig. 21. The corresponding variance response surface is plotted in Fig. 22, and its contour plot is shown in Fig. 23. 


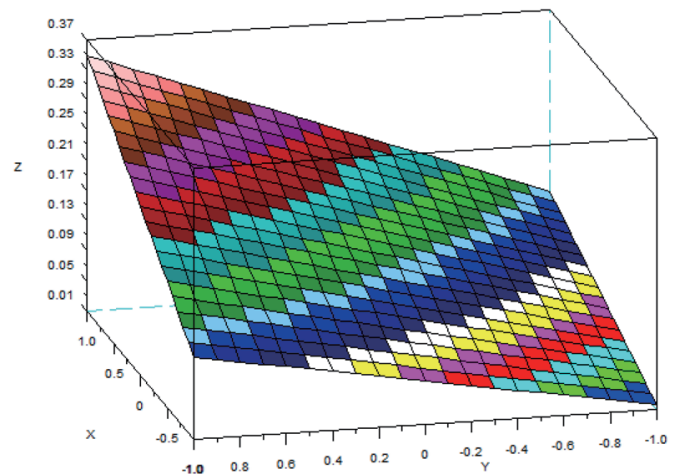

Fig. 20. (Color online) Surface plot of mean response surface.

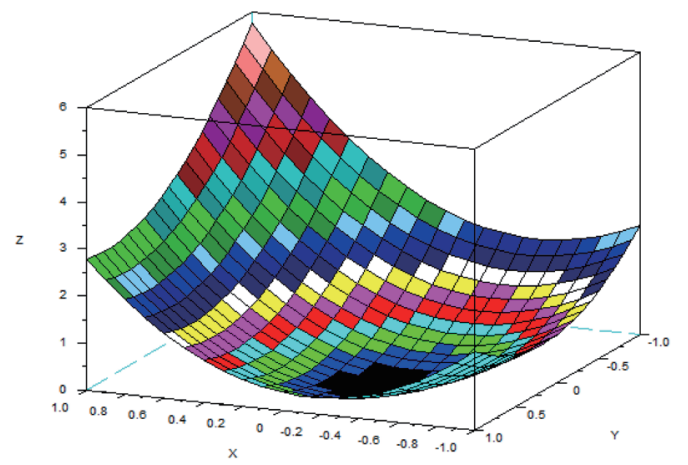

Fig. 22. (Color online) Surface plot of variance response surface.

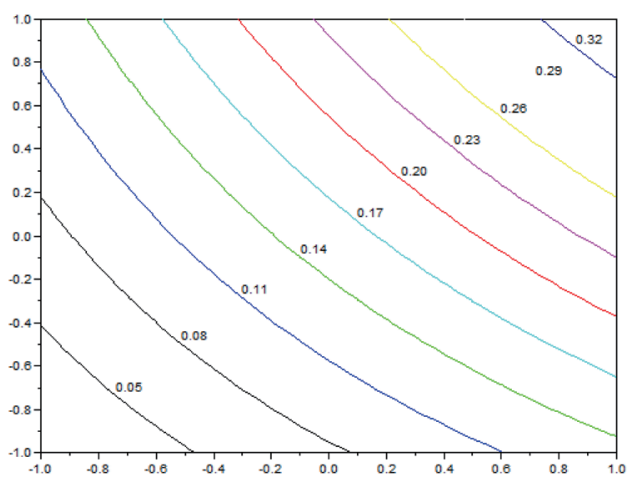

Fig. 21. (Color online) Contour plot of mean response surface.

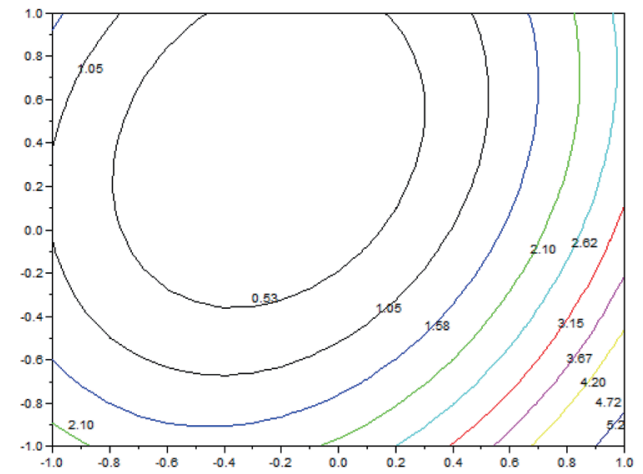

Fig. 23. (Color online) Contour plot of variance response surface.

The optimization problem is thus formulated, and the statistical model is constructed. In the following, the optimization process by the OPSO method is discussed to derive the optimal solution.

\section{Optimal Solution of Statistical Model by OPSO Method}

\subsection{OPSO derivation}

The statistical model derived by the response surface method is used in the optimization process to derive the optimal solution. However, the optimal solution may be located anywhere in the range of -1.0 and +1.0 . The local optimal solution may not be the correct optimal solution in the global region. Therefore, the OPSO process is proposed to derive the optimal solution in an efficient manner. The local and global search processes are run simultaneously. The global optimal solution can be ensured during the search for the optimal solution.

By adding random seeds into the formulation, the OPSO method can move beyond the local optimal solution if the global solution is more optimal than the local solution. In the following, the OSPO method is formulated. In the response surface method, the nonlinear problem is 
approximated as a first-order statistical model problem. However, the curvature of this firstorder model is not small. That means that the nonlinearity property is still obvious in this problem. This phenomenon affects the search process when the algorithm approaches the optimal solution.

The particle swarm optimization emulates the group dynamic behavior of animals. Each particle in a group is not only affected by individual particles, but also affected by the overall group. There are position and velocity vectors defined for each particle. The search method combines the contribution of the individual particle with that of the group. For a particle as a point in a search space with $D$-dimensions, ${ }^{(17-19)}$ the $i$-th particle associated with the problem is defined as

$$
X_{i d}=\left(x_{i 1}, x_{i 2}, \ldots, x_{i D}\right),
$$

where $d=1,2, \ldots, D$ and $i=1,2, \ldots, P S$, with $P S$ being the population size. The respective individual particle values and the group value associated with each particle $X_{i d}$ are defined as

$$
\begin{gathered}
P_{p d}=\left(p_{p 1}, p_{p 2}, \ldots, p_{p D}\right), \\
P_{g d}=\left(p_{g 1}, p_{g 2}, \ldots, p_{g D}\right) .
\end{gathered}
$$

The refreshing speed vector is defined as

$$
V_{i d}=\left(v_{i 1}, v_{i 2}, \ldots, v_{i D}\right)
$$

The refreshing position and velocity vectors are expressed as

$$
\begin{aligned}
V_{i d}^{n+1}= & V_{i d}^{n}+c_{1} \times \operatorname{rand}() \times\left(P_{p d}-X_{i d}^{n}\right) \\
& +c_{2} \times \operatorname{rand}() \times\left(P_{g d}-X_{i d}^{n}\right),
\end{aligned}
$$

where $X_{i d}^{n+1}=X_{i d}^{n}+V_{i d}^{n}$.

When the search process begins, the initial guess solution should be set first. In the iteration process, the particle is updated by the values coming from both the group and individual particle contributions. The convergence condition depends on the minimum of the average square error of the particle. Both the individual particle and group contributions are mixed together in the search process.

In the optimization problem, there might be a local minimum problem. The optimal solution might fall into a local trap whereby it cannot escape the trap. In fact, a local minimum point does not represent a global minimum solution in the entire range. By considering the group contribution, a random function can be adopted to escape the local interval. An inertia weighting factor is also considered in this algorithm to increase the convergence rate and added 
to the following expression:

$$
\begin{gathered}
V_{i d}^{n+1}=W \times V_{i d}^{n}+c_{1} \times \operatorname{rand}() \times\left(P_{p d}-X_{i d}^{n}\right)+c_{2} \times \operatorname{rand}() \times\left(P_{g d}-X_{i d}^{n}\right), \\
W=W_{\max }-\frac{W_{\text {max }}-W_{\text {min }}}{g e n_{\max }} \times \text { gen } .
\end{gathered}
$$

Here, $c_{1}$ and $c_{2}$ are both constants. $W_{\max }$ is the initial weighting value, $W_{\min }$ is the final weighting value, gen is the current generation, and gen $_{\max }$ is the number of final generations. However, the above-mentioned expression is actually a linear modification. To make the algorithm suitable for the nonlinear search problem, many nonlinear modification methods have been proposed to refresh the velocity vector. The modified term is defined as the key factor. By setting the learning factors $c_{1}$ and $c_{2}$ to be larger than 4.0, the modification in the speed vector is expressed as

$$
\begin{aligned}
& V_{i d}^{n+1}=K \times\left[\begin{array}{l}
V_{i d}^{n}+c_{1} \times \operatorname{rand}() \times\left(P_{p d}-X_{i d}^{n}\right) \\
+c_{2} \times \operatorname{rand}() \times\left(P_{g d}-X_{i d}^{n}\right)
\end{array}\right], \\
& K=\frac{2}{\left|2-\left(c_{1}+c_{2}-\sqrt{\left(c_{1}+c_{2}\right)^{2}-4 \times\left(c_{1}+c_{2}\right)}\right)\right|} .
\end{aligned}
$$

However, the modified term is complicated. In the following, a modified particle swarm optimization (PSO) method, called OPSO, is proposed to improve the search process effectively. A simpler orthogonal array in the Taguchi method is used in this algorithm to simplify the search process.

\subsection{Orthogonal array algorithm}

Before running the OPSO algorithm, two objective functions are first defined. Particle swarms are composed of an individual particle swarm $O_{i d}$ and a group swarm $A_{i d}$.

$$
\begin{gathered}
O_{i d}=X_{i d}^{n}+W V_{i d}^{n}+c_{1} \times \operatorname{rand}() \times\left(P_{p d}-X_{i d}^{n}\right) \\
A_{i d}=X_{i d}^{n}+W V_{i d}^{n}+c_{2} \times \operatorname{rand}() \times\left(P_{g d}-X_{i d}^{n}\right)
\end{gathered}
$$

The two functions are functions of three control factors in the OPSO method. Three levels are defined for the control factors. Therefore, the orthogonal array has three control factors with three levels. The $\mathrm{S} / \mathrm{N}$ ratios for SMFP and DMFBP are used in the experimental runs in 
combinational sets. Assume that the optimal solution is expressed as $Q_{i d}$. Then, $Q_{i d}$ is adopted to refresh the particle position and velocity vectors, as shown by the expression below. The particle refreshing process in OPSO is illustrated in Fig. 24.

$$
\begin{gathered}
V_{i d}^{n+1}=Q_{i d}-X_{i d}^{n} \\
X_{i d}^{n+1}=Q_{i d}
\end{gathered}
$$

\subsection{Optimal solution of statistical model}

After the above formulation for the optimization problem, the optimal solution was found. The optimal SMFP and optimal DMFBP values were found at the same time by the proposed MPCI method. The derived optimal solution could provide optimal geometric sizes for laser ablation.

By the response surface and OPSO methods, the mathematical statistical model of the studied problem was provided and verified. This model is highly useful in the associated smart machinery industrial applications. The optimal solution could be found at the ends of the entire search range, so the global solution was found instead of a local solution. Results showed that the proposed mathematical method is a better search process.

The local optimal solution is avoided and the proposed OPSO algorithm can yield the optimal solution at the endpoints globally in the entire search range. In the OPSO method, global and local particles are searched at the same time. The convergence rate of this method is higher than that of the conventional search method. The related confirmation experiments showed that the proposed methodology can provide good predictions that are in close agreement with the results of the practical experimental runs. It is verified that the proposed optimal solution obtained using the OPSO algorithm can minimize the geometric sizes in the practical laser ablation problem.

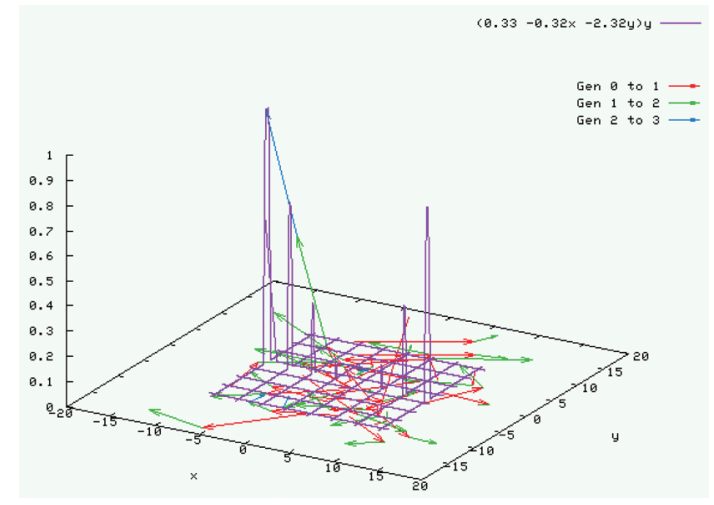

(a)

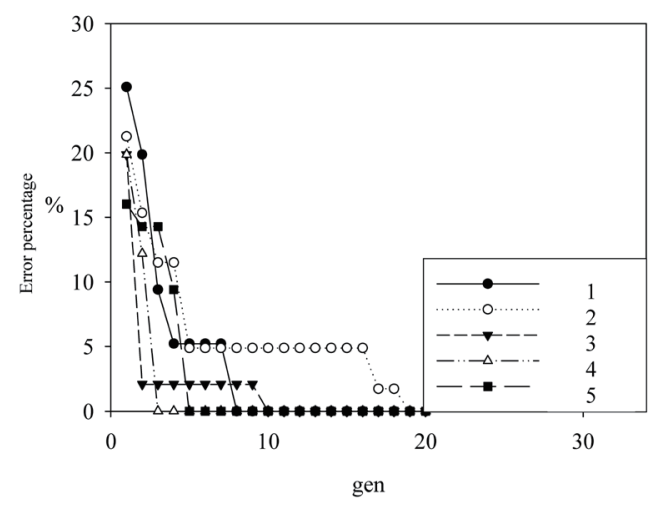

(b)

Fig. 24. (Color online) Illustrated OPSO process for optimization problem. (a) Illustrated refreshing process from first generation to third generation. (b) Illustrated error percentage from first generation to fifth generation. 
The optimal solution $\left(x_{1}, x_{2}, x_{3}\right)=(1,1,1)$ is obtained, giving the laser power of $9.5 \mathrm{~W}$, the draw step of $23 \mu \mathrm{m}$, and the step period of $25 \mu \mathrm{s}$. HD is $225.3621 \mu \mathrm{m}$ and IHD is $385.3621 \mu \mathrm{m}$.

\section{Discussion}

The results of three cases of multiobjective optimization are shown in Table 8. The total received laser energy required to perform the drilling function for hole opening is determined by three control factors: laser power, draw step, and step period. The laser power is the laser beam energy density. The draw step is the moving distance between points. The step period is the moving time between points.

From the results of Cases 1, 2, and 3, we found that the optimal solution is $\mathrm{A}_{1} \mathrm{~B}_{1} \mathrm{C}_{1}$ or $\mathrm{A}_{3} \mathrm{~B}_{3} \mathrm{C}_{3}$, where the laser power, draw step, and step period are 9.5 and $8.5 \mathrm{~W}, 23$ and $13 \mu \mathrm{m}$, and 25 and $15 \mu$ s for $A_{1} B_{1} C_{1}$ and $A_{3} B_{3} C_{3}$, respectively.

The optimal solution derived from the results of Cases 1,2, and 3 is $A_{1} B_{1} C_{1}$ or $A_{3} B_{3} C_{3}$. The average values of $A_{1} B_{1} C_{1}$ or $A_{3} B_{3} C_{3}$ are shown in Fig. 25. The nominal-the-best target value of $220 \mu \mathrm{m}$ is verified as shown in Fig. 26. The response surface method provides the required optimal parameter settings for the laser ablation machine.

The locally informed particle swarm (LIPS) method provided a distance-based concept to enhance the search ability. ${ }^{(20)}$ The distance information is determined from the data of the neighborhoods. Actually, the fuzzy MPCI method in this study involves a similar consideration between the two objective functions. The distance concept is based on the measurement of the

Table 8

Results for three cases for multiobjective optimization.

\begin{tabular}{ccc}
\hline Case 1 & Case 2 & Case 3 \\
HD & IHD & HD and IHD \\
\hline Nominal-the-best & Nominal-the-best & Nominal-the-best \\
\hline Optimal solution & Optimal solution & Optimal solution \\
$(-0.999,-0.999,-0.999)$ & $(1.0,1.0,1.0)$ & $(1.0,1.0,1.0)$ \\
\hline Optimal solution & Optimal solution & Optimal solution \\
$(8.5 \mathrm{~W}, 13 \mu \mathrm{m}, 15 \mu \mathrm{s})$ & $(9.5 \mathrm{~W}, 23 \mu \mathrm{m}, 25 \mu \mathrm{s})$ & $(9.5 \mathrm{~W}, 23 \mu \mathrm{m}, 25 \mu \mathrm{s})$ \\
\hline Objective value & Objective value & Objective values \\
$220.0698 \mu \mathrm{m}$ & $381.2645 \mu \mathrm{m}$ & $225.3621 \mu \mathrm{m}, 385.3621 \mu \mathrm{m}$ \\
\hline
\end{tabular}

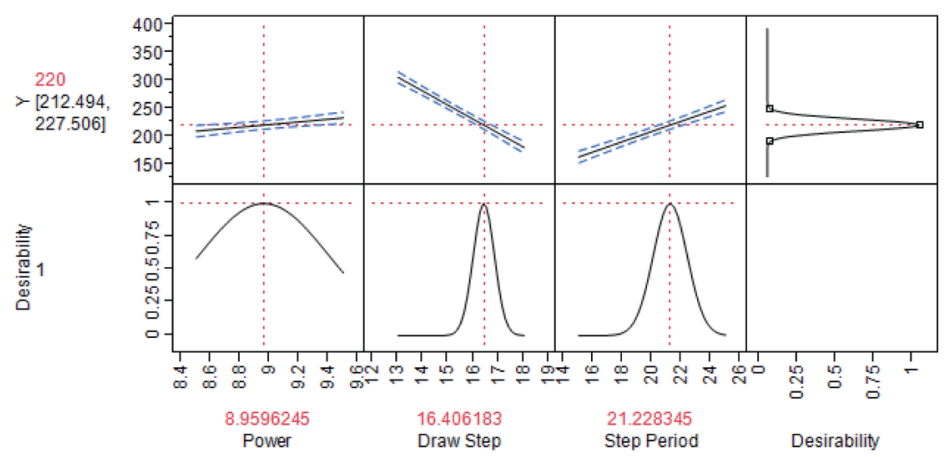

Fig. 25. (Color online) Average results of optimal values derived for Case 3. 


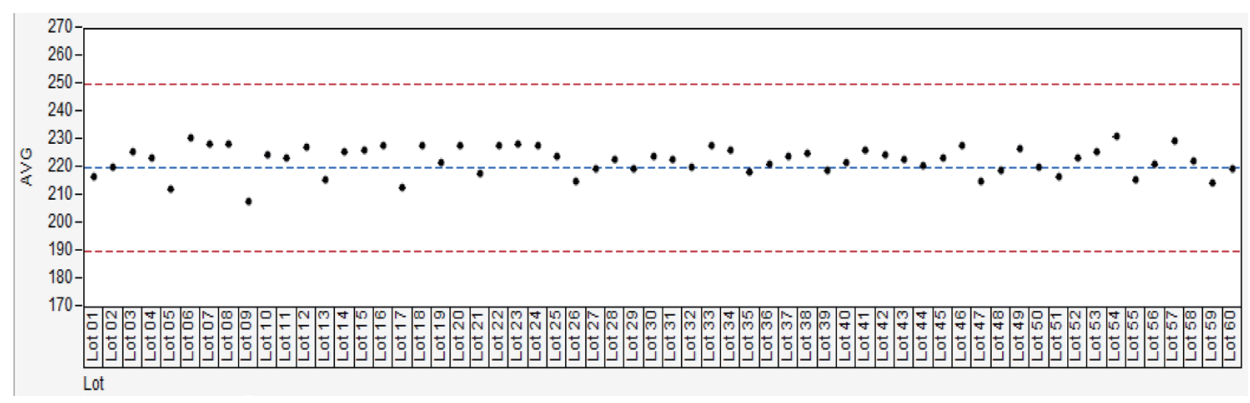

Fig. 26. (Color online) Nomimal-the-best value verification by setting optimal parameters of laser ablation machine.

data of the neighborhoods, whereas the MPCI concept is based on the measurement of fuzzy inference.

An inertia weight concept was used to enhance the performance of PSO. Three parameters, $W, c_{1}$, and $c_{2}$, are included in the speed vector. The speed vector will affect the position vector while the search process is ongoing. Instead of using $W, c_{1}$, and $c_{2}$, and related expressions from Eqs. (28) and (29), the proposed OPSO method defines the particle swarm $O_{i d}$ and group swarm $A_{i d}$. The orthogonal array method was introduced in the proposed algorithm to find the appropriate speed vector $V_{i d}$ and the next position vector $Q_{i d}$.

A maximization problem was illustrated and its results were shown in Table 1 in Ref. 19. A position vector with the multidimensional vector $X$ was defined. ${ }^{(19)}$ The search range was $[3,13]$. Results showed that OPSO yielded the result of 116.183 , which is a good approximation of 113.705 obtained by the PSO method.

The OPSO method adopts an intelligent orthogonal array method to determine the movement mechanism. The speed and position vectors are determined by the orthogonal array method instead of the conventional expression.

By setting the random seeds in the expression of speed vector, the next position vector is determined by the three parameters $W, c_{1}$, and $c_{2}$. Therefore, when the statistical model has high uncertainty, the optimization of the dual-response surface model requires an effective method to obtain the optimal value. The novelty of the OPSO method is that global and local optimal values are considered at the same time. The three parameters $W, c_{1}$, and $c_{2}$, are modified by the orthogonal array method. The optimal next position vector is specified by the Taguchi method. When the local optimal value occurs in the local range, it is possible to leave the local optimal range and continue approaching the global optimal value. The OPSO method is a form of intelligent evolutionary computation.

\section{Conclusions}

In this work, the optimal parameter design of laser ablation in the IC PoP manufacturing process was studied. The OHD and IHD were selected as two studied objective functions. Three essential geometric sizes are considered as three control factors. 
The response surface method was used to formulate the studied problem into a statistical model. To combine the two objective functions, the fuzzy-inference-based MPCI method was used to integrate the two objective functions into one compact index. To find the optimal solution for the nonlinear model, the OPSO method was used to avoid the local trap problem.

The obtained optimal solutions were verified to accomplish the parameter optimization for laser ablation. The confirmation tests of the obtained optimal solutions were performed. The developed methodology can be used to build the cloud model for laser ablation manufacturing.

The statistical model can be uploaded onto the cloud server to provide smart information for the production line. It was confirmed that the associated technology can be further used in smart manufacturing and IC packaging in the era of Industry 4.0.

\section{Acknowledgments}

The experimental setup employed in this study was partially supported by Tzan-Hsing Inc.

\section{References}

1 M. Dreiza, A. Yoshida, K. Ishibashi, and T. Maeda: Proc. 57th Electronic Components and Technology Conf. (Reno, NV, 2007) 1397-1402.

2 C. Zwenger, L. Smith, and J. Newbrough: Proc. SMTA Int. Conf. (Orlando, Florida, 2008) 17-21.

3 Institute of Printed Circuits (IPC): Acceptability of Electronic Assemblies, IPC-A-610D, A Standard Developed by IPC, February (2005). https://www.techstreet.com/hei/products/preview/1206865

4 L. M. Galantucci: CIRP Annals. 47 (1998) 141.

5 K. H. Choi, J. Meijer, T. Masuzawa, and D. H. Kim: J. Mater. Process. Technol. 149 (2004) 561.

6 J. Kim, K. Lee, D. Park, T. Hwang, K. Kim, D. Kang, J. Kim, C. Lee, C. Scanlan, C. J. Berry, C. Zwenger, L. Smith, M. Dreiza, and R. Darveaux: Proc. 58th Electronic Components and Technology Conf. (Orlando, FL, 2008) 1089-1092.

7 S. Lee: Chip Scale Review, http://ChipScaleReview.com (accessed June 2008).

8 L. Smith, M. Dreiza, and A. Yoshida: Proc. SMTA Int. Conf. (2006) 306-312.

9 K. Ishibashi: Proc. 57th Electronic Components and Technology Conf. (Reno, NV, 2007) 1403-1408.

10 J. H. Lau: Express Packaging System, Low Cost Flip Chip Technologies for DCA, WLSP and PBGA Assemblies (McGraw Hill, New York, 2000).

11 C. Zwenger and S. Lee: Proc. IMAPS Device Packaging Conf. (2009) 10-12.

12 N. C. Lee: Reflow Soldering Process and Troubleshooting: SMT, BGA, CSP and Flip Chip Technologies (Newnes Books, Oxford, 2001).

13 W. T. Silfvast: Laser Fundamentals (Cambridge University Press, Cambridge, 2008).

14 T. W. Hodapp and P. R. Fleming: J. Appl. Phys. 84 (1998) 577.

15 J. Brannon: IEEE Cir. Dev. 13 (1997) 11.

16 K. Koyamada, K. Sakai, and T. Itoh: Proc. Annu. Int. Conf. IEEE EMBS (San Francisco, CA, IEEE, 2004) 2909-2912.

17 Y. Shi and R. Eberhart: Proc. IEEE Int. Conf. Evolutionary Computation (IEEE, 1998) 69-72.

18 R. Eberhart and Y. Shi: Proc. Congr. Evolutionary Computation (2000) 84-88.

19 S. Y. Ho: IEEE Trans. Syst. Man Cybern. 38 (2008) 288.

20 B. Y. Qu, P. N. Suganthan, and S. Das: IEEE Trans. Evol. Comput. 17 (2013) 387. 